\title{
Enzymatic Resolution of Chiral Phosphinate Esters
}

\author{
Yingchun Li, Sara D. Aubert, Eugene G. Maes, and Frank M. Raushel*
}

Departments of Chemistry and of Biochemistry and Biophysics, Texas A\&M University, P. O. Box 30012, College Station, Texas 77842-3021

\section{Supporting Information}

\section{Preparation and Characterization of Compounds 1-11}

A. Compounds 1-8: Phosphinate esters were prepared through the reaction of the phosphinic chloride (1 equivalent) with the corresponding 4-substitued phenol (1 equivalent) in the presence of triethylamine (1 equivalent) at ambient temperature in dichloromethane. For characterization of these compounds, ${ }^{1} \mathrm{H}$ and ${ }^{31} \mathrm{P}$ spectra of the compounds were obtained with a Unity-300 NMR spectrometer. For ${ }^{1} \mathrm{H}$ NMR spectra, residual $\mathrm{CHCl}_{3}$ in $\mathrm{CDCl}_{3}$ was used as the reference; for ${ }^{31} \mathrm{P} \mathrm{NMR}, \mathrm{H}_{3} \mathrm{PO}_{4}$ ( $85 \%$ aqueous solution) was used as the external reference. Exact mass for these compounds were measured with a Qstar mass spectrometer. The results are listed below:

1. 4-Nitrophenyl methylphenylphosphinate (1): ${ }^{1} \mathrm{H} \mathrm{NMR}$ (in $\mathrm{CDCl}_{3}, \mathrm{ppm}$ ): 8.10-8.18 (m, 2H); 7.80-7.88 (m, 2H); 7.58-7.64 (m, 1H); 7.49-7.55 (m, 2H); 7.26-7.32 (m, $2 \mathrm{H}) ; 1.96\left(\mathrm{~d}, J_{\mathrm{P}-\mathrm{H}}=14.6 \mathrm{~Hz}, 3 \mathrm{H}\right) .{ }^{31} \mathrm{P} \mathrm{NMR}\left(\right.$ in $\left.\mathrm{CDCl}_{3}, \mathrm{ppm}\right) 45.421 . \mathrm{MH}^{+}$, Theoretical: 278.0582, Measured: 278.0585.

2. 4-Formylphenyl methylphenylphosphinate (2): ${ }^{1} \mathrm{H} \mathrm{NMR}$ (in $\mathrm{CDCl}_{3}, \mathrm{ppm}$ ): 9.89 (s, $1 \mathrm{H})$; 7.77-7.88 (m, 4H); 7.55-7.61 (m, 1H) 7.46-7.54 (m, 2H); 7.28-7.33 (m, 2H); $1.93\left(\mathrm{~d}, J_{\mathrm{P}-\mathrm{H}}=14.6 \mathrm{~Hz}, 3 \mathrm{H}\right) .{ }^{31} \mathrm{P} \mathrm{NMR}\left(\right.$ in $\left.\mathrm{CDCl}_{3}, \mathrm{ppm}\right) 44.316 . \mathrm{MH}^{+}$, Theoretical: 261.0681, Measured: 261.0698.

3. 4-Cyanophenyl methylphenylphosphinate (3): ${ }^{1} \mathrm{H} \mathrm{NMR} \mathrm{(in} \mathrm{CDCl}_{3}, \mathrm{ppm}$ ): 7.78$7.85(\mathrm{~m}, 2 \mathrm{H}) ; 7.44-7.62(\mathrm{~m}, 5 \mathrm{H}) ; 7.22-7.26(\mathrm{~m}, 2 \mathrm{H}) ; 1.91\left(\mathrm{~d}, J_{\mathrm{P}-\mathrm{H}}=14.6 \mathrm{~Hz}, 3 \mathrm{H}\right) ;{ }^{31} \mathrm{P}$ NMR (in $\mathrm{CDCl}_{3}, \mathrm{ppm}$ ) 44.789. $\mathrm{MH}^{+}$, Theoretical: 258.0684, Measured: 258.0687.

4. 4-Acetyl-phenyl methylphenylphosphinate (4): ${ }^{1} \mathrm{H} \mathrm{NMR}$ (in $\mathrm{CDCl}_{3}, \mathrm{ppm}$ ): 7.817.89 (m, 2H); 7.55-7.61 (m, 1H); 7.46-7.53 (m, 2H); 7.20-7.25 (m, 2H); 2.53 (s, 3H), 
$1.89\left(\mathrm{~d}, J_{\mathrm{P}-\mathrm{H}}=14.6 \mathrm{~Hz}, 3 \mathrm{H}\right) .{ }^{31} \mathrm{P} \mathrm{NMR}$ (in $\left.\mathrm{CDCl} 3, \mathrm{ppm}\right)$ 43.779. $\mathrm{MH}^{+}$, Theoretical: 275.0837, Measured: 275.0819.

5. 4-(Methoxycarbonyl)-phenyl methylphenylphosphinate (5): ${ }^{1} \mathrm{H} \mathrm{NMR}$ (in $\mathrm{CDCl}_{3}$, ppm): 7.92-7.96 (m, 2H); 7.81-7.89 (m, 2H); 7.45-7.60 (m, 1H); 7.46-7.53 (m, 2H); 7.19-7.24 (m, 2H); 3.87 (m, 3H); 1.91 (d, $\left.J_{\mathrm{P}-\mathrm{H}}=14.4 \mathrm{~Hz}, 3 \mathrm{H}\right){ }^{31} \mathrm{P}$ NMR (in $\mathrm{CDCl}_{3}$, ppm) 43.395. $\mathrm{MH}^{+}$, Theoretical: 291.0786, Measured: 291.0792.

6. 4-Chlorophenyl methylphenylphosphinate (6): ${ }^{1} \mathrm{H} \mathrm{NMR}$ (in $\mathrm{CDCl}_{3}$, ppm): 7.807.88 (m, 2H); 7.54-7.61(m, 1H); 7.46-7.52 (m, 2H); 7.18-7.23 (m, 2H); 7.05-7.11 $(\mathrm{m}, 2 \mathrm{H}) ; 1.87$ (d, $\left.J_{\mathrm{P}-\mathrm{H}}=14.9 \mathrm{~Hz}, 3 \mathrm{H}\right) .{ }^{31} \mathrm{P} \mathrm{NMR}$ (in $\mathrm{CDCl}_{3}, \mathrm{ppm}$ ) 43.280. $\mathrm{MH}^{+}$, Theoretical: 267.0342, Measured: 267.0333.

7. 4-Fluorophenyl methylphenylphosphinate (7): ${ }^{1} \mathrm{H} \mathrm{NMR}$ (in $\mathrm{CDCl}_{3}, \mathrm{ppm}$ ): 7.807.88 (m, 2H); 7.54-7.60 (m, 1H); 7.46-7.52 (m, 2H); 7.05-7.12 (m, 2H); 6.89-6.96 $(\mathrm{m}, 2 \mathrm{H}) ; 1.86\left(\mathrm{~d}, J_{\mathrm{P}-\mathrm{H}}=14.6 \mathrm{~Hz}, 3 \mathrm{H}\right) .{ }^{31} \mathrm{P} \mathrm{NMR}$ (in $\left.\mathrm{CDCl}_{3}, \mathrm{ppm}\right)$ 43.149. $\mathrm{MH}^{+}$, Theoretical: 251.0637, Measured: 251.0621.

8. Phenyl methylphenylphosphinate (8): ${ }^{1} \mathrm{H}$ NMR (in $\mathrm{CDCl}_{3}, \mathrm{ppm}$ ): 7.83-7.91 (m, $2 \mathrm{H})$; 7.53-7.60 (m, $1 \mathrm{H})$; 7.45-7.52 (m, 2H); 7.22-7.28 (m, 2H); 7.06-7.17 (m, $3 \mathrm{H})$; $1.86\left(\mathrm{~d}, J_{\mathrm{P}-\mathrm{H}}=14.4 \mathrm{~Hz}, 3 \mathrm{H}\right) .{ }^{31} \mathrm{P} \mathrm{NMR}\left(\right.$ in $\left.\mathrm{CDCl}_{3}, \mathrm{ppm}\right) 42.343 . \mathrm{MH}^{+}$, Theoretical: 233.0731, Measured: 233.0725.

B. Compound 10: The compound 10 was prepared following the method described in this reference: Gottfried A. Winterfeld, Yukishige Ito, Tomoya Ogawa, and Richard R. Schmidt; Eur. J. Org. Chem. (1999) 1167-1171.

C. $\left(\mathbf{R}_{\mathbf{P}} / \mathbf{S}_{\mathbf{P}}\right)-11, \mathbf{S}_{\mathbf{P}}-11$ and $\mathbf{R}_{\mathbf{P}}-11$ : To a solution of methylphenylphosphonic chloride $(1.5403 \mathrm{~g}, 8.77 \mathrm{mmol})$ in dichloromethane $(30 \mathrm{~mL})$ was added $10(2.2939 \mathrm{~g}, 8.77$ $\mathrm{mmol})$ and then triethylamine $(0.8880 \mathrm{~g}, 8.77 \mathrm{mmol})$ was added drop wise. The reaction mixture was stirred at room temperature overnight. After triethylamine hydrogen chloride was filtered, the reaction solution was condensed to dryness. The residue was then subjected to silica gel chromatography and the diasteromeric mixture $\left(\mathbf{R}_{\mathbf{P}} / \mathbf{S}_{\mathbf{P}}\right)$-11 was obtained as a colorless solid. The mixture was subjected to silica gel column chromatography and eluted with a mixture of ethyl acetate and 
hexane in a ratio of 1:4. The second diasteromer that eluted from the column was recrystallized from hexane and was determined to be the $\mathbf{R}_{\mathbf{P}} \mathbf{- 1 1}$ with X-ray crystallography. The first diastereomer eluted was therefore assigned to be $\mathbf{S}_{\mathbf{P}} \mathbf{1 1}$.

For Rp-11: ${ }^{1} \mathrm{HNMR}$ ( $\delta$ in ppm): 7.69-7.80 (m, 2H); 7.52-7.69 (m, 1H); 7.43-7.51(m, 2H), 5.75 (s, broad, $1 \mathrm{H}) ; 4.30-4.40(\mathrm{~m}, 1 \mathrm{H}) ; 4.06-4.24(\mathrm{~m}, 2 \mathrm{H}), 1.67\left(\mathrm{~d}, J_{\mathrm{P}-\mathrm{H}}=15 \mathrm{~Hz}\right.$, 3H), 1.44 (s, 9H), 1.43 (s, 9H). $\mathrm{MH}^{+}$: Measured, 400.1892 (Calculated, 400.1889).

For Sp-11: ${ }^{1} \mathrm{NMR}$ (d in ppm): 7.72-7.82 (m, 2H); 7.44-7.60 (m, 3H); 5.39 (s, broad); 4.30-4.43 (m, 2H); 3.90-4.06 (m, 1H); $1.66\left(\mathrm{~d}, J_{\mathrm{P}-\mathrm{H}}=15 \mathrm{~Hz}, 3 \mathrm{H}\right) ; 1.49$ (s, 9H); 1.40 (s, 9H). $\mathrm{MH}^{+}$: Measured, 400.1903 (Calculated, 400.1889). 
II. The Crystal Structure of $\left(R_{P}\right)-11$

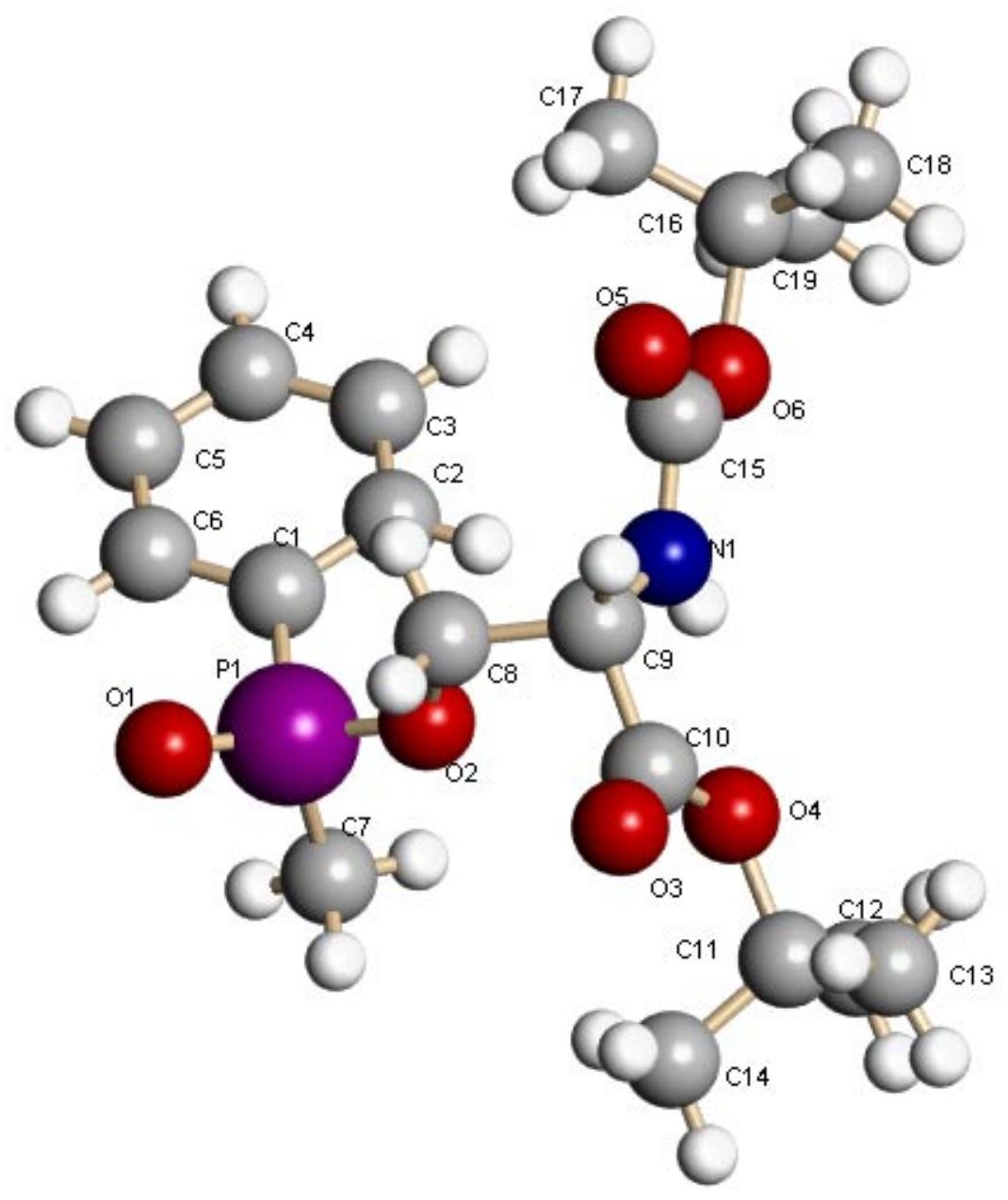

The structure of compound $\mathbf{R}_{\mathbf{P}}-\mathbf{1 1}$ 
Table 1. Crystal data and structure refinement for Rp-11.

Identification code

Empirical formula

Formula weight

Temperature

Wavelength

Crystal system

Space group

Unit cell dimensions

Volume

$\mathrm{Z}$

Density (calculated)

Absorption coefficient

$\mathrm{F}(000)$

Crystal size

Theta range for data collection

Index ranges

Reflections collected

Independent reflections

Completeness to theta $=60.65^{\circ}$

Absorption correction

Max. and min. transmission

Refinement method

Data / restraints / parameters

Goodness-of-fit on $\mathrm{F}^{2}$

Final $\mathrm{R}$ indices [I $>2 \operatorname{sigma}(\mathrm{I})]$

$\mathrm{R}$ indices (all data)

Absolute structure parameter

Largest diff. peak and hole fr10

C19 H30 N O6 P

399.41

110(2) K

$1.54178 \AA$

Monoclinic

P2(1)

$\mathrm{a}=5.758(6) \AA$

$\alpha=90^{\circ}$.

$\mathrm{b}=19.684(19) \AA$

$\beta=92.51(2)^{\circ}$.

$\mathrm{c}=8.544(8) \AA$

$\gamma=90^{\circ}$.

2

$1.371 \mathrm{Mg} / \mathrm{m}^{3}$

$1.572 \mathrm{~mm}^{-1}$

428

$0.20 \times 0.01 \times 0.01 \mathrm{~mm}^{3}$

4.49 to $60.65^{\circ}$.

$-6<=\mathrm{h}<=6,-22<=\mathrm{k}<=22,-9<=\mathrm{l}<=9$

6249

$2680[\mathrm{R}(\mathrm{int})=0.1264]$

$99.3 \%$

Semi-empirical from equivalents

0.9845 and 0.7439

Full-matrix least-squares on $\mathrm{F}^{2}$

$2680 / 1 / 244$

1.068

$\mathrm{R} 1=0.0631, \mathrm{wR} 2=0.1247$

$\mathrm{R} 1=0.1129, \mathrm{wR} 2=0.1461$

$0.06(6)$

0.527 and $-0.492 \mathrm{e} . \AA^{-3}$ 
Table 2. Atomic coordinates ( $\left.\mathrm{x} 10^{4}\right)$ and equivalent isotropic displacement parameters $\left(\AA^{2} \times 10^{3}\right)$ for Rp-11. U(eq) is defined as one third of the trace of the orthogonalized $\mathrm{U}^{\mathrm{ij}}$ tensor.

\begin{tabular}{|c|c|c|c|c|}
\hline & $\mathrm{x}$ & $\mathrm{y}$ & $\mathrm{z}$ & $\mathrm{U}(\mathrm{eq})$ \\
\hline $\mathrm{P}(1)$ & 4165(3) & $9403(1)$ & $4739(2)$ & $24(1)$ \\
\hline $\mathrm{O}(1)$ & $1714(7)$ & 9401(3) & $5066(5)$ & $22(1)$ \\
\hline $\mathrm{O}(2)$ & $5848(8)$ & $9445(3)$ & $6189(5)$ & $17(1)$ \\
\hline $\mathrm{O}(3)$ & 7043(9) & $8963(2)$ & $10002(6)$ & $27(1)$ \\
\hline $\mathrm{O}(4)$ & $9967(8)$ & $8919(2)$ & $8401(6)$ & $22(1)$ \\
\hline $\mathrm{O}(5)$ & 8926(9) & $11213(2)$ & $8446(6)$ & $27(2)$ \\
\hline $\mathrm{O}(6)$ & 11402(8) & $11015(2)$ & $6543(6)$ & $20(1)$ \\
\hline $\mathrm{N}(1)$ & $9548(10)$ & 10174(3) & $7547(7)$ & $18(2)$ \\
\hline $\mathrm{C}(1)$ & $4836(14)$ & 10064(4) & $3498(9)$ & $21(2)$ \\
\hline $\mathrm{C}(2)$ & $6996(13)$ & $10368(3)$ & $3503(9)$ & $20(2)$ \\
\hline$C(3)$ & $7464(14)$ & $10877(4)$ & $2484(10)$ & $28(2)$ \\
\hline $\mathrm{C}(4)$ & $5760(13)$ & 11073(4) & 1432(9) & $23(2)$ \\
\hline$C(5)$ & $3666(14)$ & 10781(4) & 1371(10) & $29(2)$ \\
\hline$C(6)$ & $3194(13)$ & 10273(4) & 2399(9) & $22(2)$ \\
\hline$C(7)$ & $5198(15)$ & $8669(4)$ & $3899(9)$ & $31(2)$ \\
\hline $\mathrm{C}(8)$ & $5508(12)$ & 9878(4) & 7471(8) & $23(2)$ \\
\hline $\mathrm{C}(9)$ & $7759(12)$ & 9913(4) & $8424(9)$ & $20(2)$ \\
\hline $\mathrm{C}(10)$ & 8194(13) & 9193(3) & $9026(9)$ & $20(2)$ \\
\hline$C(11)$ & $10600(14)$ & $8204(4)$ & $8743(9)$ & $22(2)$ \\
\hline $\mathrm{C}(12)$ & $12480(13)$ & 8094(4) & 7633(9) & $28(2)$ \\
\hline$C(13)$ & $11506(14)$ & $8165(4)$ & 10385(9) & $36(2)$ \\
\hline$C(14)$ & $8600(14)$ & 7760(4) & $8387(10)$ & $34(2)$ \\
\hline$C(15)$ & $9903(13)$ & $10858(4)$ & $7584(9)$ & $20(2)$ \\
\hline$C(16)$ & $12069(13)$ & 11737(3) & $6396(9)$ & $20(2)$ \\
\hline $\mathrm{C}(17)$ & $10010(13)$ & 12141(4) & $5917(10)$ & $27(2)$ \\
\hline $\mathrm{C}(18)$ & $13229(13)$ & 11959(3) & 7899(9) & $24(2)$ \\
\hline$C(19)$ & $13762(12)$ & $11700(4)$ & $5127(9)$ & $26(2)$ \\
\hline
\end{tabular}




\section{Determination of Enantiomeric Excess for the Products of Enzymatic Resolution with Chiral Electrophoresis.}

The following are electropherograms and analytical results obtained for enzymatic resolution products, $\mathbf{R p - 5}$ and $\mathbf{S p - 5}$, from hydrolysis of racemic $\mathbf{5}$ with wild type PTE and its mutant TAGW respectively. For instruments, analytical methods and procedures, see reference: Zhu, W.; Wu, F.; Raushel, F. M.; and Vigh, G. "Capillary Electrophoretic Separation of Organophosphates with a Phosphorus Stereogenic Center Using Octakis(2,3-diacetyl-6-sulfato)- $\gamma$ cyclodextrin as Resolving Agent” Journal of Chromatography A, 2000, 895, 247 254.

\section{Rp-5 From Enzymatic Resolution with Wild type PTE}

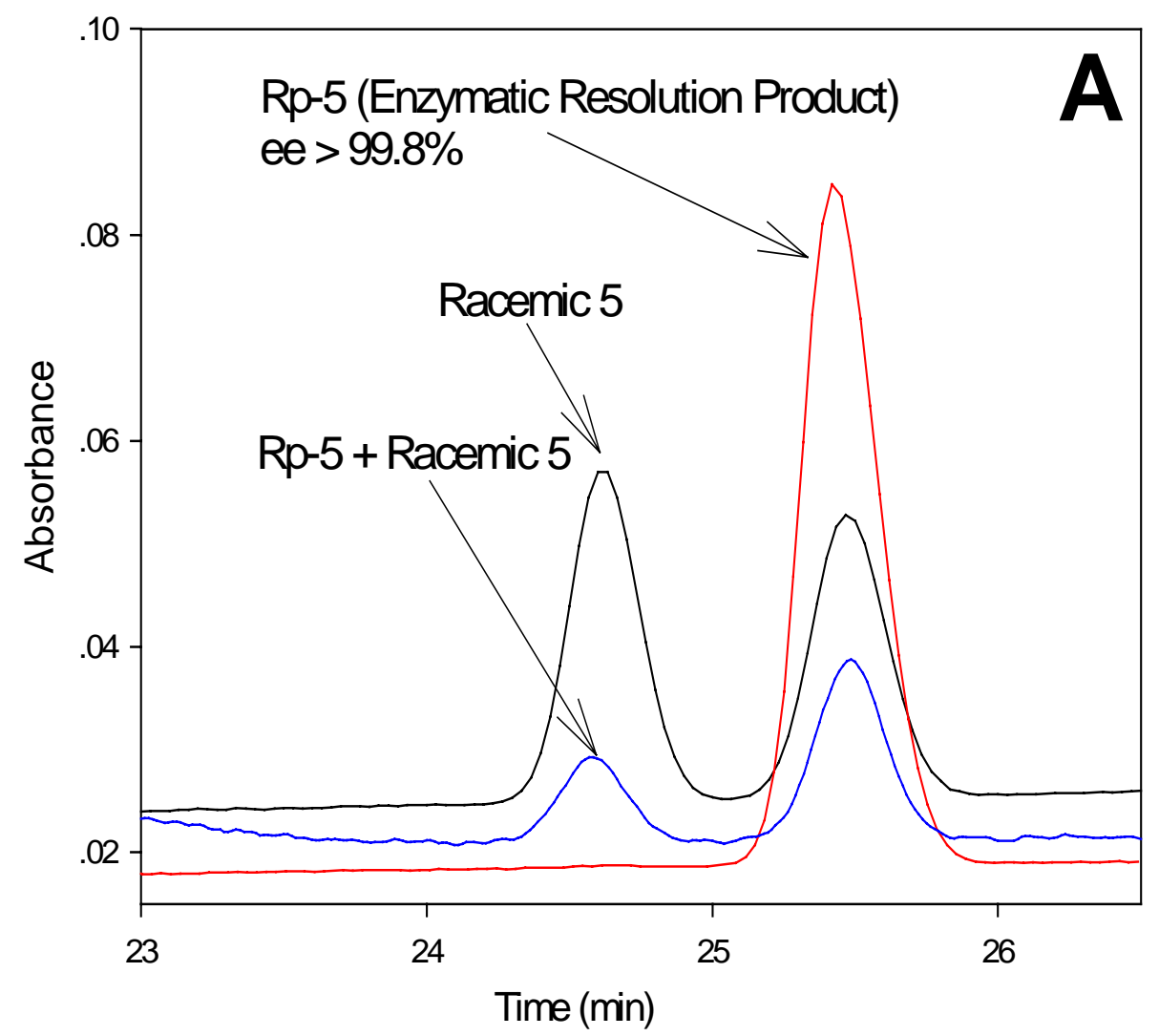


Sp-5 from Enzymatic Resolution with TAGW (Mutant of PTE)

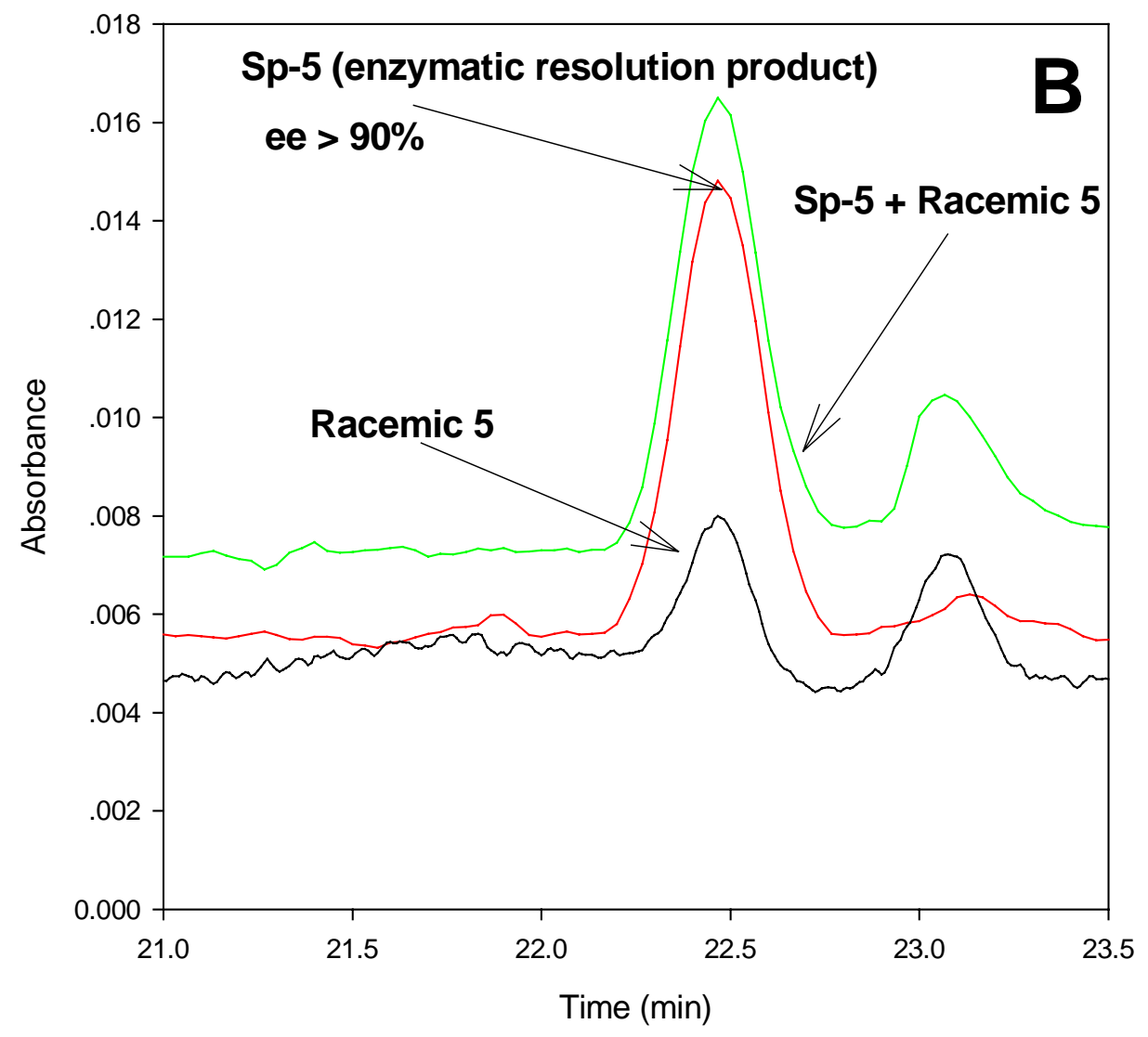

